\title{
A novel Salmonella strain inactivated by a regulated autolysis system and expressing the B subunit of Shiga toxin 2e efficiently elicits immune responses and confers protection against virulent Stx2e-producing Escherichia coli
}

Gayeon Won ${ }^{\dagger}$, Tae Hoon $\mathrm{Kim}^{\dagger}$ and John Hwa Lee

\begin{abstract}
Background: Salmonella Typhimurium (S. Typhimurium) inactivated by a regulated autolysis system was genetically engineered to express the homo-pentameric B subunit of Shiga toxin 2e (Stx2eB) on its surface. To prepare a strain able to yield autolyzed Salmonella bearing Stx2eB, the plasmid pJHL184 harboring stx $x_{2 e B}$ gene was transformed into the attenuated S. Typhimurium strain, JOL1454. Stx2eB subcloned into the antigen delivery cassette of the plasmid was expressed as fusion protein with the outer membrane protein

Results: The expression of Stx2eB fused to the signal peptide in JOL1454 was validated by immunoblot analysis. To determine the immunogenicity of JOL1454, female BALB/c mice were intramuscularly injected with $1 \times 10^{8}$ CFU of the inactivated cells at weeks 0 and 2. Significantly elevated levels of IgG and lgA specific to Stx2eB was observed at weeks 4 and 6 post-immunization (PI) $(P<0.05)$. Proportion of $\mathrm{CD}^{+} \mathrm{CD}^{+} \mathrm{T}$ lymphocyte subpopulation was also significantly augmented in in vivo stimulated splenocytes relative to that in the control group. The increased titers of $\operatorname{lgG} 1$ and $\operatorname{lgG} 2 \mathrm{a}$, and of immunomodulatory cytokines indicated that the immunization elicited Th1 and Th2 immune responses. Further, immunomodulatory cytokine genes (IL-6, IL-17A, IL21 and JOL 1454) efficiently upregulated in naïve porcine peripheral blood mononuclear cells (PBMCs) pulsed with JOL1454. At week $6 \mathrm{Pl}$, following the challenge with a virulent Stx2e-producing Escherichia coli in the mice, all immunized mice survived whereas approximately $30 \%$ of the mice in the control group died.
\end{abstract}

Conclusions: JOL1454 provided superior immunogenicity and effective protection against challenge with a sublethal dose, which demonstrates its potential as a candidate vaccine against edema disease.

Keywords: Shiga toxin, Edema disease, Bacterial ghost, Salmonella typhimurium

\footnotetext{
* Correspondence: johnhlee@jbnu.ac.kr

${ }^{\dagger}$ Equal contributors

College of Veterinary Medicine, Chonbuk National University, Iksan campus,

Gobong-ro 79, Iksan 54596, Republic of Korea
} 


\section{Background}

Among Shiga toxin-producing Escherichia coli (STEC) strains, Stx2e is the second most common subtype of $s t x_{2}$ found in isolates from environmental sources [1]. Although STEC harboring the stx2e gene has only rarely been detected in human feces, causing mild diarrhea [2], stx $2 e$ is the most frequently encountered variant of stx gene in STEC isolated from porcine feces [3], and STEC harboring the stx2e gene has been reported to contribute to the virulence of edema disease (ED) in weaned piglets [4]. Shiga toxin 2e, encoded by the stx2e gene, inhibits protein biosynthesis by ribosome inactivation, which is chiefly responsible for the clinical signs and lesions of $E D$, including subcutaneous and submucosal edema. In addition, brain vascular injury caused by endothelial cell edema can elicit fatal neurological disorders and sudden deaths [4]. Binding of the non-toxic pentamer B subunits of Stx2e (Stx2eB) to the cell surface globotetraosylceramide (Gb4Cer) receptor allows the toxic A subunit of Stx2e (Stx2eA) to enter the cytoplasm, where cytotoxic effects occur [5]. Prevention of Stx2eB binding to the relevant receptors located on the intestines and cerebral endothelial cells thus represents a possible mechanism to prevent the transmission of this pathogen. Hence, Stx2eB may represent a practical target for the generation of neutralizing antibodies that could contribute to impairing the interaction between Stx2eB and cell surface receptors on intestines, thereby inhibiting the subsequent cytotoxic effects on epithelial cells that are mediated by Shiga toxin [5].

ED causes significant economic losses due to sudden deaths of infected pigs. The factors affecting the prevalence of ED are not clearly understood, despite mortality rates due to ED as high as 50 to $90 \%$ [6], with substantial variance among countries and farming units, and depending on the health status of the infected pigs. Elaborate efforts have been made in an attempt to reduce disease burden and economic loss in the swine industry. In particular, the need for optimal vaccination strategies against ED has increased, as frequently reported incidences of antimicrobial-resistant STEC in swine farms worldwide become progressively more burdensome to public health [7]. In addition, the administration of antibiotics appears to come too late to treat diseased pigs, since even when antibiotics are administered at the onset of visible clinical signs, severe neurological symptoms subsequently develop. Accordingly, several vaccine strategies based on targeting Shiga toxin have arisen. Active and passive immunization of piglets with an Stx2e toxoid has been reported to provide protection against challenge with Stx2e toxin [6]. However, those results did not imply that the toxoid would protect piglets against virulent STEC infection. Live attenuated STEC carrying genetically modified Stx2e has been constructed that confers somewhat effective protection against a challenge with a lethal dose [6], although a high degree of reactogenicity remained. Thus, despite the continuous effort to improve vaccines against ED, no commercial vaccine is currently available.

A virulent strain of Salmonella Typhimurium has been successfully prepared for use in expressing a broad range of homologous antigens, to induce enhanced immune responses against them [8]. To minimize the risk of live attenuated Salmonella reverting to a virulent strain, autolyzed ghost strains derived from Salmonella enterica, including S. Enteritis [9], S. Gallinarum [10], and S. Typhi [11] have been genetically engineered for use as candidate vaccines or vaccine delivery carriers. Inactivated bacterial ghosts retaining the entire surface antigenic features of the original bacteria can efficiently target antigen-presenting cells and induce strong immunological responses [12].

A particular form of autolyzed bacteria generated via PhiX174 E gene-mediated lysis, so-called "bacterial ghosts" (BGs), are non-living gram-negative bacterial cell envelopes that lack cytoplasmic contents, yet conserve all the surface components of their parental bacteria $[12,13]$. BGs have induced strong immunological immune responses against retained surface antigenic determinants, such as lipopolysaccharide and peptidoglycan [14]. The capacity of BGs as a presentation system for heterologous antigens has been evaluated in previous studies $[15,16]$. Foreign target proteins presented by BGs have been successfully expressed as outer membrane proteins via fusion with signal sequences, or been translocated into the periplasmic space [16]. Salmonella ghost strains have been widely used as vehicles for antigen delivery due to their ability to induce adjuvant effects by invading host immune systems [15]. In the present study, an attenuated $S$. Typhimurium strain harboring a recombinant plasmid carrying a lysis gene permitting induction of bacterial autolysis, as well as the $s t x_{2 e B}$ gene, was constructed. Lysis of $S$. Typhimurium was mediated by the PhiX174 E gene under the control of the faceto-face promoter system to generate $S$. Typhimurium ghost cells [17]. During lysis, Stx2eB protein fused with the outer membrane protein A signal peptide (ompA ss) is expressed, which enables Stx2eB to be exported across membranes of autolyzed cells. The immunogenicity of $S$. Typhimurium ghosts expressing Stx2eB was evaluated in a mouse model, and protective efficacy was also examined by challenging immunized mice with virulent STEC.

\section{Methods}

Bacterial strains and culture conditions

All bacteria strains and plasmids used in this study are described in Table 1 . The $S$. Typhimurium mutant 
Table 1 Bacterial strains and plasmids used in this study

\begin{tabular}{|c|c|c|}
\hline Strain/plasmid & Description & $\begin{array}{l}\text { Reference/ } \\
\text { source }\end{array}$ \\
\hline \multicolumn{3}{|l|}{ Bacterial strains } \\
\hline \multicolumn{3}{|l|}{ E. coli } \\
\hline BL21(DE3)pLysS & 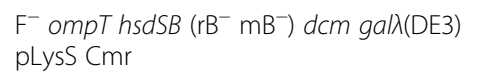 & Promega \\
\hline JOL232 & $\begin{array}{l}\mathrm{F}^{-} \lambda^{-} \varphi 80 \Delta(\text { lacZYA-argF }) \text { endA1 recA1 } \\
\text { hadR17 deoR thi-1 glnV44 gyrA96 relA1 } \\
\text { DasdA4 }\end{array}$ & Lab stock \\
\hline JOL606 & $\begin{array}{l}\text { Wild-type } \mathrm{LT}^{+}, \mathrm{K}^{+} 9^{+}, \mathrm{FG}^{+}, \mathrm{F} 8^{+}, \mathrm{stx}_{2}^{+}, \mathrm{st} x_{2 e}^{+} \\
\text {STEC isolate from pig }\end{array}$ & Lab stock \\
\hline JOL654 & $\begin{array}{l}\text { Wild-type } \mathrm{LT}^{+}, \mathrm{F}_{1} 8^{+}, \mathrm{STa}^{+}, s t x_{2}^{+}, s t x_{2 e}^{+} \mathrm{STEC} \\
\text { isolate from pig }\end{array}$ & Lab stock \\
\hline \multicolumn{3}{|l|}{ S. Typhimurium } \\
\hline JOL912 & $\begin{array}{l}\Delta / o n \Delta c p x R \Delta a s d \text {, a derivative of } \mathrm{S} \text {. } \\
\text { Typhimurium }\end{array}$ & [20] \\
\hline JOL 1400 & JOL912 harboring pJHL184 & This study \\
\hline JOL 1454 & JOL912 harboring pJHL184-stx $x_{2 e B}$ & This study \\
\hline \multicolumn{3}{|l|}{ Plasmids } \\
\hline pET28a & IPTG-inducible expression vector; $\mathrm{Km}^{\mathrm{r}}$ & Novagen \\
\hline pET28a-stx $2 e B$ & pET28a derivative containing $s t x_{2 e B}$ & This study \\
\hline pJHL184 & $\begin{array}{l}\text { asd }^{+} \text {vector, pBR ori plasmid carrying ss } \\
\text { ompA/His }, \text { multiple cloning site, cl857/ } \\
\lambda \text { PR promoter, araC } P_{\text {araBAD, }} \text { phiX } 174 \text { lysis } \\
\text { gene } E\end{array}$ & {$[47]$} \\
\hline pJHL184-stx $x_{2 e B}$ & pJHL184 harboring stx 2 2eB gene & This study \\
\hline
\end{tabular}

strains, $\Delta a s d$ lon $\Delta c p x R$ JOL912 and $\Delta a s d$ Escherichia coli $\mathrm{X} 6212$, were grown in either Luria-Bertani (LB) broth or LB agar at $37{ }^{\circ} \mathrm{C}$ with $50 \mu \mathrm{g} / \mathrm{ml}$ of diaminopimelic acid (DAP) (Sigma-Aldrich, St. Louis, MO). The bacterial strains harboring the ghost plasmid were grown at $28{ }^{\circ} \mathrm{C}$ in nutrient broth $(\mathrm{NB})$ containing $0.2 \% \mathrm{~L}$-arabinose. All bacterial strains were stored at $-80{ }^{\circ} \mathrm{C}$ in growth medium containing $20 \%$ glycerol.

\section{Construction of Salmonella ghosts bearing Stx2eB}

The $S$. Typhimurium mutant strain $\Delta$ asd $\Delta$ lon $\Delta c p x R$, JOL912, was prepared by allelic exchange methods as previously described [18]. The ghost plasmid, pJHL184, carries a pBR origin, a multiple cloning site (MCS), a Cterminal His-tag, and a ghost cassette [19]. The $s t x_{2 e B}$ gene was amplified by polymerase chain reaction (PCR) from wild-type STEC JOL606 isolated from pig diarrhea, using the primer pair, S1: 5'-ccgccaattcaagaagatgtttatggcg-3' and S2: 5'-ccgcaagcttgtcattattaaactgcac-3'. The thermal cycle parameters of the PCR reaction consisted of an initial denaturation at $94{ }^{\circ} \mathrm{C}$ for 5 mins, followed by 30 cycles of $95{ }^{\circ} \mathrm{C}$ for $15 \mathrm{~s}, 54{ }^{\circ} \mathrm{C}$ for $15 \mathrm{~s}$, and $72{ }^{\circ} \mathrm{C}$ for $30 \mathrm{~s}$, with a final extension step of 7 mins at $72{ }^{\circ} \mathrm{C}$. The gene fragment was designed to produce an EcoRI restriction endonuclease site at the $5^{\prime}$ end and a HindIII site at the 3' end. The resultant PCR products were subcloned into the overexpression plasmid pET28a, thus generating pET28a-stx $2 e B$. The E. coli BL21 (DE3) pLysS strain was transformed with pET28a-stx $2 e B$. Stx2eB protein was purified as described previously [19]. The $s t x_{2 e B}$ gene-containing DNA fragments derived from pET28a$s_{2} x_{2 e B}$ were subcloned into EcoRI/HindIII-digested pJHL184 to generate the recombinant plasmid pJHL184$s_{2 e B}$ [17]. Stx $2 \mathrm{eB}$ secretion into the periplasmic space is achieved by fusion with the signal sequence of $E$. coli outer membrane protein OmpA, which was subcloned into the MCS of pJHL184. pJHL184-stx $x_{2 e B}$ was initially transformed into E. coli $\times 6212$ (JOL232) to maintain the stability of the plasmid in the absence of antibiotics, and the plasmid subsequently was introduced into JOL912 by electroporation. The resultant strain was designated as JOL1454. The $\Delta$ asd $\Delta l o n \Delta c p x R \quad S$. Typhimurium strain JOL1400, carrying pJHL184, was used as a vector control.

\section{Production of Salmonella ghosts bearing Stx2eB}

A single colony of JOL1454 was inoculated into nutrient broth containing $0.2 \% \mathrm{~L}$-arabinose, and the inoculum was incubated at $28{ }^{\circ} \mathrm{C}$ with agitation at $120 \mathrm{rpm}$ until mid-logarithmic growth phase to achieve mass production of the strain. The cells were collected and washed twice with nutrient broth (NB) to remove Larabinose. The cells were resuspended in $100 \mathrm{ml} \mathrm{NB}$ and incubated at $42{ }^{\circ} \mathrm{C}$ in a shaking incubator at $200 \mathrm{rpm}$ to induce $E$ gene-mediated lysis over the course of $48 \mathrm{~h}$. After lysis, the ghost cells were harvested via centrifugation at $13,000 \mathrm{rpm}$ for $15 \mathrm{~min}$, washed twice with sterile phosphate-buffered saline (PBS) (pH 7.4), and stored at $-70{ }^{\circ} \mathrm{C}$.

\section{Stx2eB expression in JOL1454}

Western blot analysis was performed to verify the expression of Stx $2 \mathrm{eB}$ antigen from JOL1454, as previously described [19]. Bacterial ghosts expressing the target antigen, and hyperimmune rabbit serum raised against Stx2eB, were prepared as previously described [19]. Protein lysates of the prepared ghost cells $(5 \mu \mathrm{l})$ were subjected to sodium dodecyl sulfate-polyacrylamide gel electrophoresis (SDS-PAGE) on 15\% gels. Resolved proteins were transferred onto polyvinylidene fluoride membranes (Millipore, Billerica, MA, USA), immunoblot analysis was performed, and immunoreactive bands were detected as previously described using hyperimmune rabbit serum raised against Stx2eB $(1: 5,000)$ and a HRP-labeled goat anti-rabbit $\operatorname{IgG}(1: 8,000)$ [19]. JOL1400, JOL912 carrying pJHL184 and the purified Stx2eB protein were utilized as negative and positive controls, respectively. Subsequently, the amount of Stx2eB expressed in JOL1454 was relatively quantified in a calibration standard using indirect enzyme-linked 
immunosorbent assays (ELISA) [21]. Stx2eB containing $6 \times$ His-tag at the C-terminal end in JOL1454 were purified by Ni-NTA spin columns (Ni-NTA Spin Kit, Qiagen) according to the manufacturer's instruction. The His-tagged Stx2eB protein in a volume of $5 \mathrm{ml}$ of the prepared ghost cell $\left(1 \times 10^{8} \mathrm{cfu}\right.$ per $\left.\mathrm{ml}\right)$ were eluted from the column with $2 \mathrm{ml}$ of elution buffer $(7 \mathrm{M}$ urea: $7 \mathrm{M}$ urea; $0.1 \mathrm{M}$ sodium dihydrogen phosphate; $0.01 \mathrm{M}$ Tris. $\mathrm{Cl}$; $\mathrm{pH}$ 8.0). A calibration standard was generated by using twofold serial dilutions of Stx2eB protein extracted from BL21 harboring pET28a-stx $2 e B$. The diluted protein samples ranging from $1 \mu \mathrm{g}$ to $31.25 \mathrm{ng}$, and the purified His-tagged protein as antigen were coated in the ELISA plate (Greiner) and incubated at $4{ }^{\circ} \mathrm{C}$ overnight. After the incubation, the hyperimmune rabbit serum raised against Stx2eB (1:300) and a HRP-labeled goat anti-rabbit IgG $(1: 5,000)$ were used as primary and secondary antibodies, respectively as previously described [19]. The concentration of the His-tagged Stx2eB protein expressed in JOL1454 were calculated by the known concentration of the purified Stx2eB corresponding to values of optical density at $490 \mathrm{~nm}$ in the calibration standard. The final concentration of the protein presented was based on the number of cells in initial bacterial culture.

\section{Animal experiments}

Eighteen female BALB/c mice were randomly assigned to two groups at five weeks of age. The mice in group $\mathrm{A}$ were intramuscularly immunized with $1 \times 10^{8}$ JOL1454 ghost cells at weeks zero and two. PBS was injected into the mice in group B, which served as a non-immunized control group. For measurement of total serum immunoglobulin (Ig) G, as well as of IgG1 and IgG2a, serum samples were collected, and for measurement of secretory IgA (sIgA), vaginal wash samples were collected, as previously described [20], at weeks $0,2,4$ and 6. All samples were stored at $-70{ }^{\circ} \mathrm{C}$ until used. For immunological assays using the primed splenocytes, additional 10 mice (5 mice per group) were inoculated at week 0 using the same protocol described above. Further, although ED results from oral transmission of the pathogen in natural infections, we found that ED did not occur in BALB/C model mice orally challenged with JOL654. However, intraperitoneal injection with the challenge strain efficiently induced symptoms in mice similar to those of ED such as hemorrhage in small intestine and bloody diarrhea. At week 7, non-immunized and immunized mice were intraperitoneally challenged with wild type STEC JOL654 isolated from porcine diarrhea (Table 1). The murine sublethal dose $\left(2 \times 10^{7} \mathrm{CFU}\right)$ was determined by the Reed-Muench method [22] following a protocol used in a previous study [10]. Mice were monitored daily for mortality, clinical signs, and body weight for one week after inoculation.

\section{Humoral and cellular immunological response}

Stx2eB-specific titers of total IgG, the IgG1 and $\operatorname{IgG} 2 \mathrm{a}$ isotypes, and secretory $\operatorname{IgA}(\operatorname{IgA})$ in sera or vaginal washes, as appropriate, were measured by ELISA [19] on 96-well plates with $500 \mathrm{ng}$ of purified Stx2eB protein coating each well. Fluorescence-activated cell sorting (FACS) was used to assay changes in $\mathrm{T}$ cell subpopulations induced by immunization. Splenocytes were aseptically isolated from mice at day seven postimmunization, and single-cell suspensions were prepared. One million cells were stained by combining them with anti-mouse CD3a-PE and anti-mouse CD4perCP-vio700 antibodies (Miltenyi Biotec, Bergisch Gladbach, Germany) and incubating for $20 \mathrm{~min}$. After washing twice with FACS buffer (Miltenyi Biotec, Bergisch Gladbach, Germany), changes in $\mathrm{CD}^{+}$and $\mathrm{CD} 4$ ${ }^{+} \mathrm{T}$ cell subpopulations were determined using a MACSQuant ${ }^{\odot}$ analyzer (Miltenyi Biotec, Bergisch Gladbach, Germany). Splenocyte proliferation following antigen stimulation in vitro was assessed by incorporation of MTT (3-(4,5-dimethylthiazol-2-yl)-2,5diphenyltetrazolium bromide), which is only converted to blue formazan dye by actively proliferating cells [23]. Splenocytes were isolated from the immunized group at week two post-immunization (PI). Suspensions of $1 \times 10^{6}$ single cells were cultured in triplicate with $500 \mathrm{ng} / \mathrm{ml}$ of stx2eB antigen in RPMI 1640 medium (GIBCO, cat. no.11875093) containing 5\% FCS (GIBCO, cat. no. 10099141), at $37{ }^{\circ} \mathrm{C}$ in a $5 \%$ $\mathrm{CO}_{2}$ incubator for $72 \mathrm{~h}$. Following stimulation, the cells were incubated with MTT $(1 \mathrm{mg} / \mathrm{ml})$ for another $4 \mathrm{~h}$. The precipitated blue formazan in each well was solubilized in dimethyl sulfoxide, and colorimetric absorbance was measured as optical density (OD) at $570 \mathrm{~nm}$ with a spectrophotometer. The results are presented as a stimulation index, calculated as the mean OD value of the wells stimulated with antigen, divided by the mean OD value of the unstimulated wells.

\section{Cytokine measurement}

Induction of expression at the mRNA level of the cytokines, interleukin-4 (IL-4) and interferon- $\gamma$ (IFN- $\gamma$ ), induced by immunization of the mice was measured by reverse transcription real-time PCR. At week 2 PI, splenocytes were isolated from immunized and nonimmunized mice. Viable cells $\left(1 \times 10^{6}\right)$ stimulated with $500 \mathrm{ng} / \mathrm{ml}$ of Stx $2 \mathrm{eB}$ protein were incubated in a 96-well cell culture plate with RPMI 1640 medium supplemented with $20 \%$ fetal bovine serum (FBS) in a humidified $5 \% \mathrm{CO}_{2}$ atmosphere for $72 \mathrm{~h}$. Total RNA from the cultured cell suspensions was isolated using a GeneAll ${ }^{\circ}$ 
Hybrid-R kit (GeneAll Biotechnology, Seoul, Korea) and converted into cDNA with a ReverTra Ace ${ }^{\circ}$ qPCR RT Kit (FSQ-101, TOYOBO, Japan). Levels of IL-4 and IFN$\gamma$ mRNA expression were quantified by real-time reverse transcription polymerase chain reaction (RT-PCR). The sequences of the primer pairs used to amplify IL-4, IFN$\gamma$ and $\beta$-actin (used as an internal standard) were those described by Lut et al. [24]. The mRNA expression levels of the cytokines were determined by the threshold method, using Cycle threshold $\left(\Delta C_{\mathrm{T}}\right)$ values calculated based on the internal standard. The fold change of the mRNA levels compared to those of the non-immunized group is expressed as $2^{-(\Delta \Delta C T)}$ [25].

\section{Cytokine profiles assessed in porcine PBMC pulsed with JOL1454}

To determine the extent of porcine lymphocyte activation following stimulation of the lysed $S$. Typhimurium expressing Stx2eB, immunomodulatory cytokines were measured in mRNA level in naïve porcine peripheral blood mononuclear cells (PBMC) pulsed in vitro with JOL1454. Naïve porcine PBMCs were obtained from $2 \mathrm{ml}$ of blood samples of five non-vaccinated pigs (15$18 \mathrm{~kg}, 6$ weeks of age) raised at an experimental animal farm of the college by density gradient centrifugation using Histopaque- $1077^{\circ}$ solution (Sigma, St. Louis, MO) following the manufacturer's instruction. After the PBMC isolation, the cells were placed in RPMI 1640 medium supplemented with $1 \%$ fetal bovine serum and $1 \%$ glutamine/penicillin/streptomycin (Thermo Scientific), and then plated at a density of $5 \times 10^{6}$ per well in 96 well cell culture plate. Subsequently, the resuspended cells were treated with the lysed JOL1454 with 10 multiplicity of an infection (MOI), and were incubated for $48 \mathrm{~h}$ at $37{ }^{\circ} \mathrm{C}$ in a humidified atmosphere of $5 \% \mathrm{CO}_{2}$. Following the incubation, the pulsed cells were lysed directly in 500ul of Trizol reagent for RNA extraction. The preparation of RNA and cDNA were carried out as described above and $2 \mu \mathrm{l}$ synthesized cDNA were added as qRT-PCR template. Cytokine (IL-6, IL-17A, IL-21, IFN- $\gamma$ ) mRNA expressions were determined by using the primer pairs [26-28] with $\mathrm{SYBR}^{\circ}$ Green Realtime PCR Master Mix (QPK-201, TOYOBO, Japan) following the manufacturer's instruction. Porcine glyceraldehyde-3phosphate dehydrogenase (GADPH) and 60s ribosomal protein L19 (RPL-19) genes were used as reference genes for normalization [29]. $\Delta C_{\mathrm{T}}$ values were standardized by the average $\mathrm{Ct}$ values of two internal controls and nonstimulated cells. Relative fold changes were determined by $2^{-\Delta \Delta \mathrm{CT}}$ method [25].

\section{Statistical analysis}

Data are presented as mean \pm standard deviation (s.d.). One-way ANOVA test was used to evaluate significant differences in immune responses between the immunized and non-immunized groups. Statistical differences were considered significant when $P$ values were $<0.05$.

\section{Results}

Construction of Salmonella ghosts expressing Stx2eB

The Stx2eB gene was cloned under control of a constitutive promoter in the pJHL184 plasmid, which contains a convergent promoter system tightly regulating the induction of the $E$ lysis gene and consequent lysis of JOL1454. To constitutively express $s t x_{2 e B}$, JOL1454 cells were grown at $42{ }^{\circ} \mathrm{C}$ for $48 \mathrm{~h}$ in the absence of $\mathrm{L}$ arabinose. Lysis of JOL1454 was confirmed by the inviability of cells plated from JOL1454 cultures grown at $42{ }^{\circ} \mathrm{C}$. No viable cells were observed on LB plates after overnight incubation under conditions wherein the $E$ gene was repressed (data not shown). The Stx2eB protein fused to ompA expressed in JOL1454 was verified by western blot analysis. The distinct immuno-reactive band of Stx2eB fused with OmpA was observed at $\sim 24 \mathrm{kDa}$ (Fig. 1; lane 1). Considering that the size of Stx2eB protein is $\sim 13 \mathrm{kDa}$, we speculated that OmpA ss were properly fused with the target protein. In the lane 1 of Fig. 1 in which the lysed JOL1400 was loaded, the band corresponding to the size of $\mathrm{Stx} 2 \mathrm{eB}$ was not shown. For the positive control, Stx2eB fused to $6 \mathrm{xHis}$ purified from pET28a-stx2eB in BL21 was loaded where immuno-reactive band was detected (Fig. 1; lane 3). The relative amount of Stx2eB protein expressed in JOL1454 was estimated by the linear regression standard curve showing a fit of $R^{2}=0.971$. Concentration of the His-

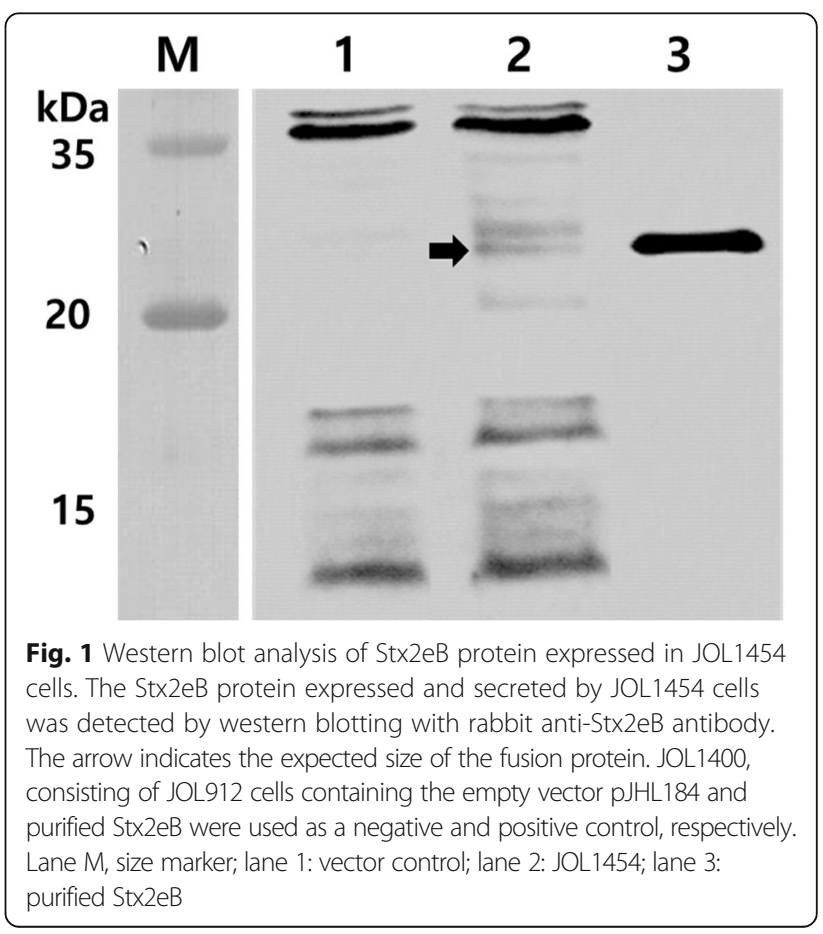


tagged Stx2eB was approximately $909.18 \mathrm{ng}$ per $10^{8} \mathrm{cfu}$ of JOL1454.

\section{Humoral and mucosal immune responses raised by immunization}

The immunogenicity induced by JOL1454 immunization of the mice was assessed by measuring Stx2eB-specific serum IgG and sIgA titers. The sIgA and IgG titers specific to the Stx2eB antigen were significantly elevated $(P<0.05)$ in the JOL1454 group compared to those in the control group (Fig. 2). The IgG titers significantly increased at week $4(P<0.05,2.59$-fold $)$ and week 6 postimmunization (PI) $(P<0.001,2.79$-fold $)$ compared to those measured in the negative control. The concentration of SIgA was moderately increased at week 2 PI and was significantly raised in comparison to those in the negative control at week 4 and 6 PI $(P<0.05)$. Furthermore, the ratio of the IgG1 to IgG2a (IgG1/IgG2a) immunoglobulin isotypes, markers of T helper 2 (Th2) and $\mathrm{T}$ helper1 (Th1) lymphocytes, respectively, was calculated from the titers of the individual isotypes. JOL1454 immunization induced IgG1 to a greater degree than it

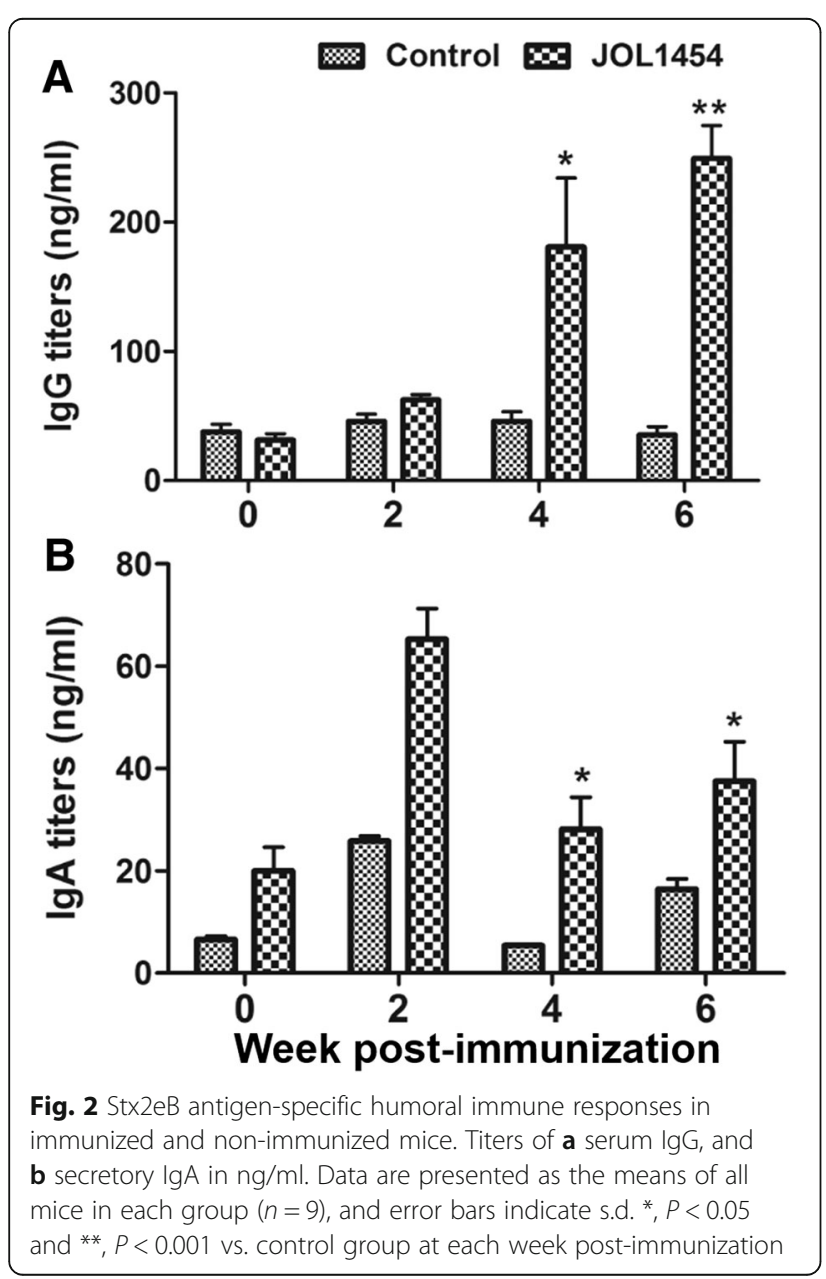

did IgG2a (Fig. 3). The level of IgG1 was consistently higher than that of IgG2a throughout the entire observation period (Fig. 3).

\section{T lymphocyte proliferation induced by immunization}

Changes in splenic $\mathrm{T}$ cell fractions at week one postimmunization were assessed by measuring expression of the $\mathrm{T}$ cell surface markers CD3 and CD4 using flow cytometry. Both the $\mathrm{CD}^{+} \mathrm{T}$ cell population and $\mathrm{CD}^{+} \mathrm{CD}^{+} \mathrm{T}$ cell subpopulations were significantly elevated $(P<0.05$, respectively) in JOL1454-immunized mice (Fig. 4a), leading to significant average increases of $3.52 \%$ and $2.6 \%$ in the $\mathrm{CD}^{+}$and $\mathrm{CD}^{+} \mathrm{CD}^{+}{ }^{+} \mathrm{T}$ cell subpopulations, respectively, compared to the nonimmunized group. In the MTT cell viability assay, the JOL1454-immunized mice showed a significant increase $(P<0.05)$ in stimulation index $(\mathrm{SI})$ at week 2 $\mathrm{PI}$, resulting in a significantly increased mean SI of $2.54 \pm 0.49$ in the immunized group, compared to $1.26 \pm 0.29$ in the PBS control group (Fig. $4 b$ ).

\section{Cytokine measurement}

To evaluate immunomodulatory cytokines secreted by the stimulated splenic lymphocytes, mRNA copy numbers of the IL-4 and IFN- $\gamma$ cytokines were analyzed by qPCR. Fold changes were normalized against the murine $\beta$-actin gene. In vitro stimulation of splenic $\mathrm{T}$ cells with JOL1454 resulted in marked changes in relative fold values for the IFN- $\gamma(P$ $<0.05)$ and IL-4 $(P=0.01)$ cytokines in the immunized group compared to those in the control group (Fig. 5). These results indicated that the expression of IL-4 and IFN- $\gamma$ in the immunized group were significantly upregulated compared to the control group, following stimulation of splenic $\mathrm{T}$ cells with Stx2eB.

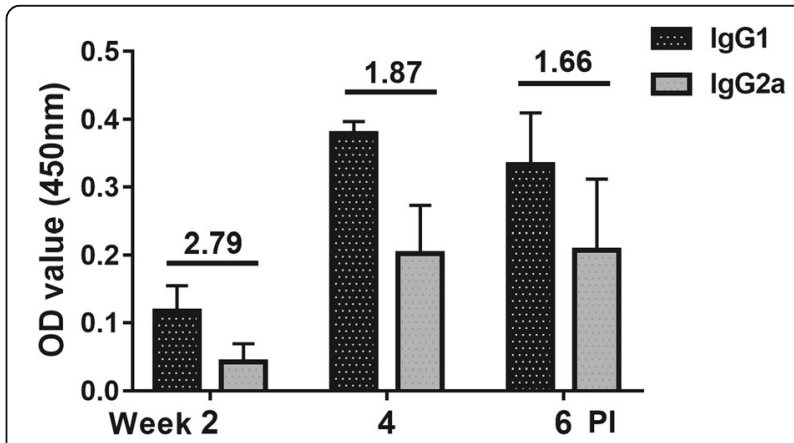

Fig. 3 The levels of two $\lg G$ isotypes, $\lg G 1$ and $\lg G 2 a$, elicited by JOL1454 immunization. The values indicate the means of optical density at $450 \mathrm{~nm}$ from sera collected from immunized mice $(n=9)$, and error bars indicate standard deviation (s.d). The numbers above the bars indicate the lgG1/lgG2a ratios. PI: post-immunization 

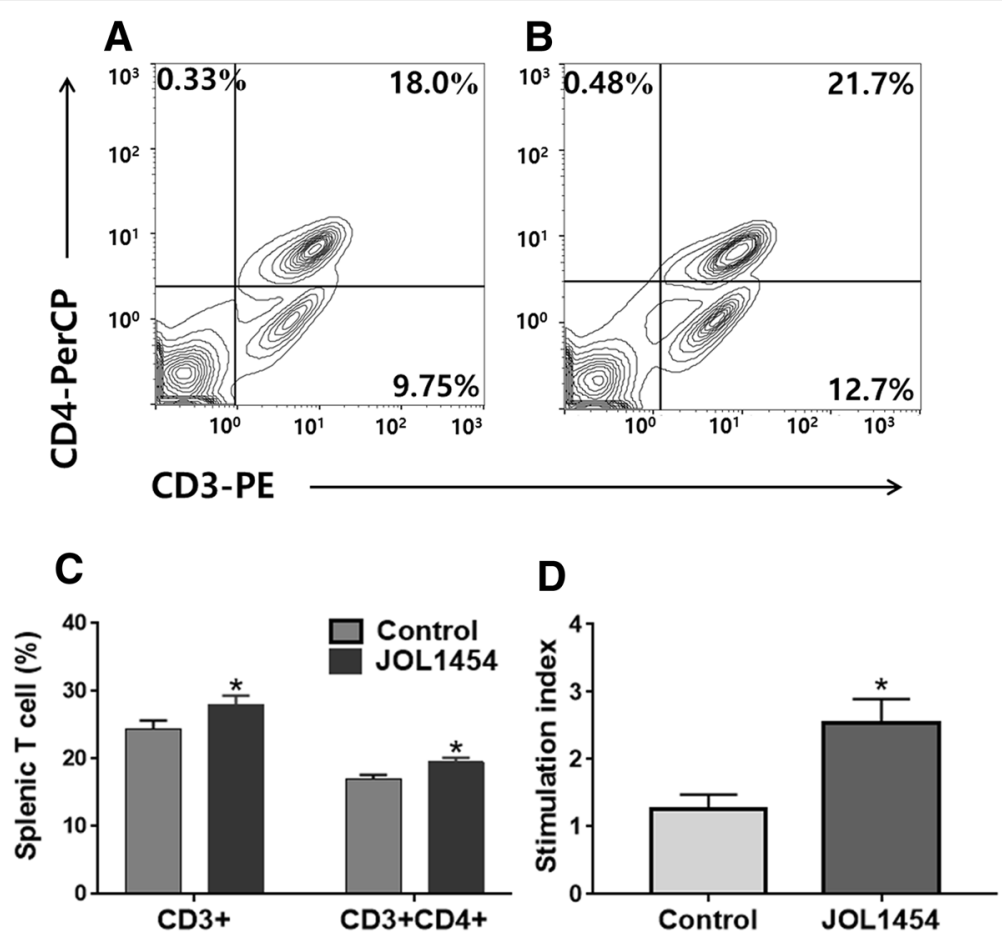

Fig. $4 \mathrm{~T}$ cell-related immune responses elicited in JOL1454-immunized mice. Representative flow cytometry scatter dot plots for CD3+ and CD3 + CD4+ splenic T cell populations of non-immunized mice (a) and the immunized mice (b). The subpopulations are presented as a percentage of gated cells. CD3+ and CD3 + CD4+ T lymphocyte populations in immunized and non-immunized mice (c) and stimulation index (SI) values of splenic T cells of the purified Stx2eB protein-immunized group, determined by MTT assay (d). The values are expressed as the mean \pm s.d. of five individual values. ${ }^{*}, P<0.05$

Immunomodulatory cytokines measured in the activated porcine PBMC

In vitro stimulation of porcine PBMCs with JOL1454 induced upregulation of immunomodulatory cytokine gene expressions (Fig. 6). The expression of IFN- $\gamma$, one of

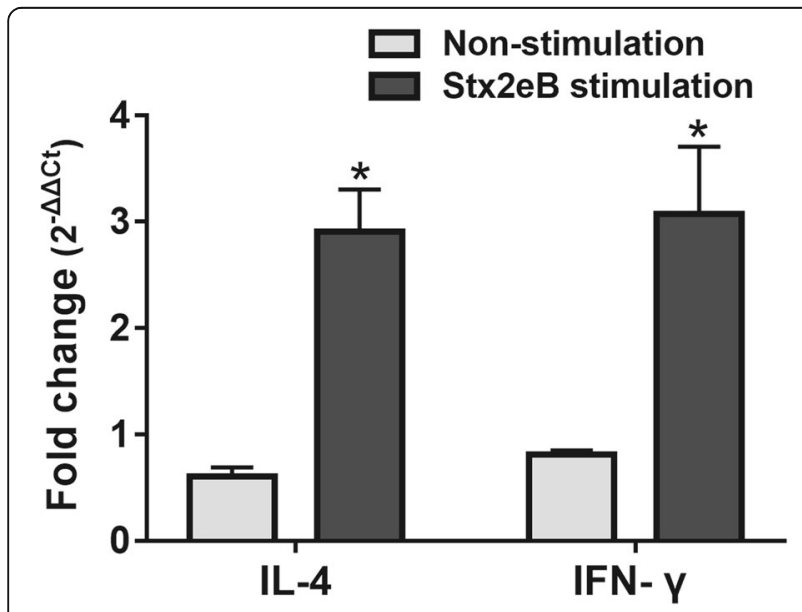

Fig. 5 Cytokine mRNA transcript levels in stimulated and unstimulated splenic T cells isolated from JOL1454-immunized mice. The mRNA transcript levels of cytokines were evaluated by performing RT q-PCR with gene-specific primers. Each fold change value represents the mean \pm standard error of the mean (SEM) of five individual values. ${ }^{*} P<0.05$ when values were compared with the control group
Th1-specific cytokine [29] and IL-6 promoting Th2 responses [30] increased up to $28.9 \pm 8.5$ and $15.13 \pm$ 3.7 fold, respectively. Gene expression of IL-17A involving with differentiation of Th17 cells [31], were predominantly upregulated in the stimulated PBMC (71.88 \pm 12.29 -fold). Concomitantly, a moderate increase of expression of IL-21 produced by Th17 cells $[32,33](6.07 \pm 1.73$-fold $)$ were also observed in the pulsed PBMCs.

\section{Protective efficacy conferred by JOL 1454}

To assess the protective efficacy conferred by JOL1454 immunization, all immunized mice were intraperitoneally injected with a sublethal dose of virulent STEC JOL654 at week 7 PI. The survival rates and weight losses of the mice were monitored in immunized and non-immunized animals for seven days after challenge. In both the immunized and the control group mice, weight loss, which began only following the challenge, was observed through day 1 post-challenge. While the weights of the immunized animals rapidly recovered, body weight of the negative control was markedly dropped compared to the immunized mice at days 2, 3, 4 and 7 post challenge $(P<0.05)$ (Fig. $7 \mathrm{~b})$. All immunized mice survived during the entire observation period, but $22.2 \%$ of the non-immunized mice died within $18 \mathrm{~h}$ of 


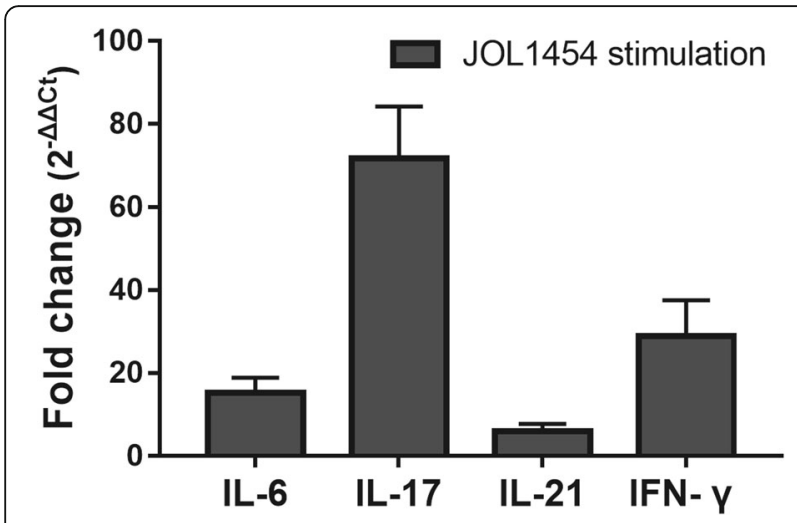

Fig. 6 The mRNA expression of cytokines evaluated in in vitro stimulated porcine PBMC. The immunomodulatory cytokines were measured in mRNA level by using qRT PCR. Relative fold changes were calculated based on $2^{-\triangle \Delta C T}$ method. Data are presented with the mean $\pm \operatorname{SEM}(n=5)$

challenge (Fig. 7a). Clinical signs such as diarrhea, hunched posture, and hair election were also observed in non-immunized mice.

\section{Discussion}

Stx2eB is not only an intrinsically non-toxic and immunogenic protein of STEC but also a proven candidate vaccine antigen for prevention and control of ED in pigs [34]. In this study, we constructed an autolyzed $S$. Typhimurium strain expressing Stx2eB, which we designate as JOL1454, and confirmed the expression of Stx2eB fused with the six transmembrane domains (TMD) of the E. coli outer membrane protein A (OmpA) in this strain (Fig. 1). OmpA signal peptides have been successfully employed to translocate target antigens across the cytoplasmic space or insert proteins into the outer membranes of gram-negative bacteria [35]. In the present study, detection of Stx $2 \mathrm{eB}$ protein expression in the cell pellet indicated that the OmpA signal peptide efficiently directed the expressed Stx2eB in the Salmonella ghosts (Fig. 1). Additionally, the relative amount of the His-tagged Stx2eB protein was measured in the lysed JOL1454. The significantly elevated levels of antibodies specific to Stx2eB in mice immunized with JOL1454 (Fig. 2) demonstrated that modified $S$. Typhimurium mediated efficient expression of Stx $2 \mathrm{eB}$ on the outer membrane of the Salmonella ghosts.

In this study, immunization of mice with JOL1454 markedly elevated their titers of anti-Stx $2 \mathrm{eB} \operatorname{IgG}$ and sIgA (Fig. 2). The in vivo role of systemic IgG (sIgG) in neutralizing Shiga toxin has been emphasized in strategies to protect piglets against ED [36]. Stx-specific sIgG antibodies can protect immunized pigs against systemic infection with STEC, and the magnitude of protection relies on the dose of the antibodies [37]. sIgA antibodies,
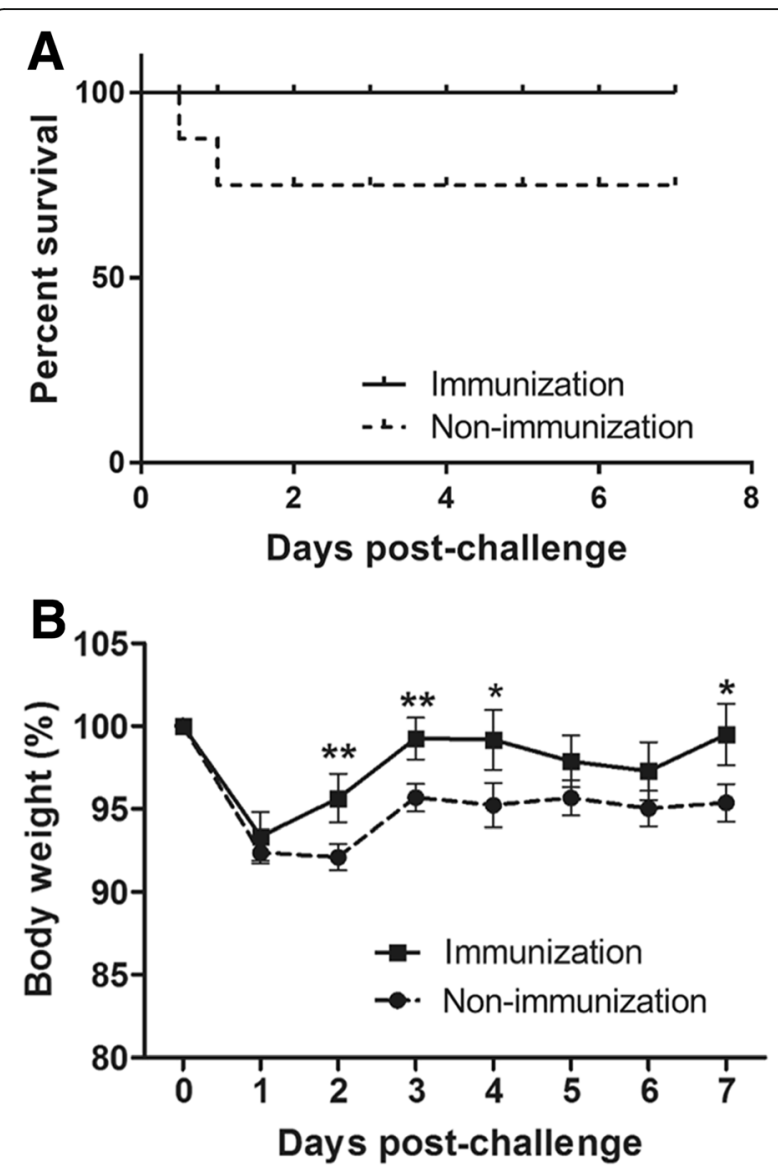

Fig. 7 Protective efficacy against challenge with a sublethal dose of JOL654 conferred by JOL1454. a Survival rates of immunized and non-immunized mice after challenge. $\mathbf{b}$ Percentage (\%) in mouse body weight. Each point represents the mean of 9 mice per group, and error bars indicate s.d. ${ }^{*} P<0.05$ or ${ }^{* *} P<0.01$ when values were compared with the control group

however, serve as the first line of defense against infection at mucosal surfaces [38]. As the natural infection of STEC occurs via the oral route, sIgA also has an essential role in preventing adherence or attachment of pathogens [39]. These findings indicate that immunization with JOL1454 may mediate protection against ED by eliciting humoral and mucosal immune responses against Stx2eB.

The IgG isotypes, IgG1 and IgG2a, are regarded as markers of $\mathrm{T}$ helper (Th) 2- and Th1-type immune responses, respectively [40]. The Fc portions of the IgG1 and IgG2a antibodies primarily interact with the activatory $\mathrm{F}_{\mathrm{C}-} \gamma$ receptors $\left(\mathrm{F}_{\mathrm{C}} \mathrm{R} \mathrm{R}\right)$ III and $\mathrm{F}_{\mathrm{C}} \mathrm{R} \mathrm{R}$ IV, which elicit Th2- and Th1-type immune responses, respectively [41]. The IgG1-to-IgG2a (IgG1/IgG2a) ratio has been used to determine the relative contribution of Th2- versus Th1type immune responses to STEC infection [42]. In our current study, the IgG1/IgG2a ratio declined from 2.79 to 1.66 in response to JOL1454 immunization (Fig. 3). 
The decreased ratio indicated that the contribution of IgG2a antibodies continuously increased during the observation period (Fig. 3). This implies that, despite the predominance of the Th2 subpopulation, the Th1 immune response was steadily elevated in JOL1454immunized animals.

Naïve CD4 + T lymphocytes stimulated with an antigenic peptide differentiate into Th1 and Th2 subpopulations [43]. Th1 and Th2 cells promote cell-mediated immune responses involving antigen-specific cytotoxic effects and humoral immune responses, respectively [44]. JOL1454 immunization significantly elevated the $\mathrm{CD}^{+} \mathrm{CD} 4^{+} \mathrm{T}$ cell subpopulations in mice, (Fig. 4a), indicating that JOL1454 can stimulate naive $\mathrm{CD} 4^{+} \mathrm{T}$ cell maturation. Additionally, we observed that the copy numbers of IL-4 and IFN- $\gamma$ mRNA were significantly elevated in restimulated splenic lymphocytes of the immunized mice (Fig. 5). The immunomodulatory cytokines, IFN- $\gamma$ and IL-4, are exclusively expressed in mature Th1 and Th2 cells, respectively [43]. Thus, this observation supports the interpretation that JOL1454 was able to efficiently stimulate CD4+ T cell differentiation into Th1 and Th2 subpopulations, which drive the induction of cellular and humoral immune responses, respectively.

Salmonella ghosts elicit robust cell-mediated immune responses (CMI), as they retain the entire surface antigenic determinants of their pre-lysed condition, in the native forms thereof [10]. We observed markedly enhanced in vitro SI in re-stimulated splenic $\mathrm{T}$ cells of JOL1454-immunized mice (Fig. 4B). This implies that JOL1454 can induce lymphocyte proliferation, which is a primary parameter of CMI responses. In parallel, mRNA levels of IFN- $\gamma$, a marker of CMI, were significantly elevated in splenic $\mathrm{T}$ cells after in vitro restimulation. In a previous report, mice immunized with purified Stx2eB protein did not show increased serum levels of IFN- $\gamma$ [36]. These results support the conclusion that the Salmonella ghost system delivering Stx2eB can induce CMI responses in immunized mice.

Ren et al. constructed a plasmid expressing Stx $2 \mathrm{eB}$ for use as a candidate vaccine, and investigated its protective efficacy using the construct in a mouse model [45]. In that study, immunized mice were intraperitoneally challenged with a virulent ED-associated E. coli, and $80 \%$ of the immunized mice survived. Immunization of mice with JOL1454 in our study also offered highly effective protection against a challenge with wild-type STEC. While no mortality occurred in any of the immunized mice, approximately $30 \%$ of the mice in the nonimmunized group died. The protection conferred by JOL1454 immunization might be due to the following: JOL1454 Salmonella ghosts efficiently secreted Stx2eB in the cells due to fusion of the protein with ompA ss;
Stx2eB delivered by JOL1454 ghosts elicited circulating antibodies specific to Stx2eB, which may neutralize Shiga toxin; and Salmonella ghosts bearing Stx2eB can trigger CMI responses mediating phagocytosis and elimination of the challenge strain. Additionally, JOL1454 efficiently induced upregulated expression of immunomodulatory cytokines involved with activation of Th1, Th2 and Th17 cells in the pulsed porcine PBMC (Fig. 6), which implicated that JOL1454 may have a capacity to differentiate naïve porcine lymphocytes toward matured $\mathrm{CD}^{+} \mathrm{T}$ cell subpopulation. Particularly, the increased expression of IL-6, IL-17A and IL-21 in the present study indicated Th17 cells was matured and activated in the pulsed porcine lymphocytes, which is crucial for intestinal mucosal host defense [46]. These preliminary data supported that JOL1454 has a potential to elicit subsequent immune responses following the cytokine production in porcine.

\section{Conclusions}

The present data indicate that Stx2eB delivered by a Salmonella ghost strain could effectively stimulate the immune system so as to confer sufficient protection to prevent ED. The results also suggest that Salmonella inactivated by $E$ gene-mediated lysis that preserves their intact antigenic determinants may not only effectively deliver the target antigen but also provide an adjuvant property. Additionally, given the immunostimulatory effect of JOL1454 which can elicit immunomodulatory and proinflammatory cytokines in the porcine lymphocyte, this autolyzed Salmonella strain producing Stx2eB may constitute a novel alternative approach to developing an effective vaccine candidate against porcine edema disease.

\section{Abbreviations \\ BGs: Bacterial ghosts; $C_{T}$ : Cycle threshold; DAP: Diaminopimelic acid; ED: Edema disease; ELISA: Enzyme-linked immunosorbent assays; \\ FACS: Fluorescence-activated cell sorting; Gb4Cer: Globotetraosylceramide receptor; Ig: Immunoglobulin; IL: Interleukin; MCS: A multiple cloning site; MTT: 3-(4,5-dimethylthiazol-2-yl)-2,5-diphenyltetrazolium bromide; NB: Nutrient broth; OD: Optical density; ompA ss: Outer membrane protein A signal peptide; PBMCs: Peripheral blood mononuclear cells; PBS: Phosphate-buffered saline; PCR: Polymerase chain reaction; PI: Post-immunization; S. Typhimurium: Salmonella Typhimurium; SDS- PAGE: Sodium dodecyl sulfate-polyacrylamide gel electrophoresis; STEC: Shiga toxin-producing Escherichia coli; Stx2eA: Toxic A subunit of Stx2e; Stx2eB: Homo-pentameric B subunit of Shiga toxin 2e}

\section{Acknowledgements}

Not applicable.

\section{Funding}

This work was supported by the National Research Foundation of Korea (NRF) grant funded by the Korea government (MISP) (No.

2015R1A2A1A14001011).

Availability of data and materials

The datasets analysed during the current study available from the corresponding author on reasonable request. 


\section{Authors' contributions}

GW and TK conducted the experiments and was involved in manuscript preparation. JHL conceived the study, precipitated in the design of the study and was involved in manuscript preparation. All authors read and approved the final manuscript.

\section{Competing interests}

The authors declare that they have no competing interests.

\section{Consent for publication}

Not applicable.

\section{Ethics approval}

All animal experimentation work was approved by the Chonbuk National University Animal Ethics Committee (CBNU2015-00085), and was carried out according to the guidelines of the Korean Council on Animal Care and Korean Animal Protection Law, 2007; Article 13 (Experiments with Animals).

Received: 17 July 2016 Accepted: 26 January 2017

Published online: 01 February 2017

\section{References}

1. Sonntag AK, Bielaszewska M, Mellmann A, Dierksen N, Schierack P, Wieler $\mathrm{LH}$, Schmidt MA, Karch H. Shiga toxin 2e-producing Escherichia coli isolates from humans and pigs differ in their virulence profiles and interactions with intestinal epithelial cells. Appl Environ Microbiol. 2005;71(12):8855-63.

2. Pierard D, Huyghens L, Lauwers S, Lior H. Diarrhoea associated with Escherichia coli producing porcine oedema disease verotoxin. Lancet. 1991; 338(8769):762.

3. Fratamico PM, Bagi LK, Bush EJ, Solow BT. Prevalence and characterization of Shiga toxin-producing Escherichia coli in swine feces recovered in the national animal health monitoring System's swine 2000 study. Appl Environ Microbiol. 2004:70(12):7173-8.

4. Marques L, Peiris J, Cryz S, O'brien A. Escherichia coli strains isolated from pigs with edema disease produce a variant of Shiga-like toxin II. FEMS Microbiol Lett. 1987;44(1):33-8.

5. Donohue-Rolfe A, Kondova I, Mukherjee J, Chios K, Hutto D, Tzipori S. Antibody-based protection of gnotobiotic piglets infected with Escherichia coli O157:H7 against systemic complications associated with Shiga toxin 2. Infect Immun. 1999;67(7):3645-8.

6. Makino S, Watarai M, Tabuchi H, Shirahata T, Furuoka H, Kobayashi $Y$, Takeda Y. Genetically modified Shiga toxin 2e (Stx2e) producing Escherichia coli is a vaccine candidate for porcine edema disease. Microb Pathog. 2001;31(1):1-8

7. Ho WS, Tan LK, Ooi PT, Yeo CC, Thong KL. Prevalence and characterization of verotoxigenic-Escherichia coli isolates from pigs in Malaysia. BMC Vet Res. 2013;9:109-6148-9-109.

8. Russmann H, Shams H, Poblete F, Fu Y, Galan JE, Donis RO. Delivery of epitopes by the Salmonella type III secretion system for vaccine development. Science. 1998;281(5376):565-8.

9. $\quad$ Peng W, Si W, Yin L, Liu H, Yu S, Liu S, Wang C, Chang Y, Zhang Z, Hu S. Salmonella enteritidis ghost vaccine induces effective protection against lethal challenge in specific-pathogen-free chicks. Immunobiology. 2011; 216(5):558-65

10. Chaudhari AA, Jawale CV, Kim SW, Lee JH. Construction of a Salmonella gallinarum ghost as a novel inactivated vaccine candidate and its protective efficacy against fowl typhoid in chickens. Vet Res. 2012;43(1):44-54.

11. Wen J, Yang $Y$, Zhao G, Tong $S$, Yu H, Jin X, Du L, Jiang S, Kou Z, Zhou Y. Salmonella typhi Ty21a bacterial ghost vector augments HIV-1 gp140 DNA vaccine-induced peripheral and mucosal antibody responses via TLR4 pathway. Vaccine. 2012;30(39):5733-9.

12. Lubitz W. Bacterial ghosts as carrier and targeting systems. Expert Opin Biol Ther. 2001;1(5):765-71.

13. Szostak MP, Hensel A, Eko FO, Klein R, Auer T, Mader H, Haslberger A, Bunka S, Wanner G, Lubitz W. Bacterial ghosts: non-living candidate vaccines. J Biotechnol. 1996:44(1):161-70.

14. Haslberger A, Kohl G, Felnerova D, Mayr U, Fürst-Ladani S, Lubitz W. Activation, stimulation and uptake of bacterial ghosts in antigen presenting cells. J Biotechnol. 2000;83(1):57-66.
15. Jalava K, Eko FO, Riedmann E, Lubitz W. Bacterial ghosts as carrier and targeting systems for mucosal antigen delivery. Expert Rev Vaccines. 2003; 2(1):45-51.

16. Walcher P, Mayr UB, Azimpour-Tabrizi C, Eko FO, Jechlinger W, Mayrhofer $P$, Alefantis T, Mujer CV, DelVecchio VG, Lubitz W. Antigen discovery and delivery of subunit vaccines by nonliving bacterial ghost vectors. Expert Rev Vaccines. 2004;3(6):681-91.

17. Jawale CV, Kim SW, Lee JH. Tightly regulated bacteriolysis for production of empty Salmonella enteritidis envelope. Vet Microbiol. 2014;169(3):179-87.

18. Kang HY, Srinivasan J, Curtiss R. 3rd: immune responses to recombinant pneumococcal PspA antigen delivered by live attenuated Salmonella enterica serovar typhimurium vaccine. Infect Immun. 2002;70(4):1739-49.

19. Jawale CV, Lee JH. Salmonella enterica serovar enteritidis ghosts carrying the escherichia coli heat-labile enterotoxin B subunit are capable of inducing enhanced protective immune responses. Clin Vaccine Immunol. 2014;21(6):799-807.

20. Hur J, Lee JH. Immune responses to new vaccine candidates constructed by a live attenuated Salmonella typhimurium delivery system expressing Escherichia coli F4, F5, F6, F41 and intimin adhesin antigens in a murine model. J Vet Med Sci. 2011;73(10):1265-73.

21. Nemchinov LG, Natilla A. Transient expression of the ectodomain of matrix protein 2 (M2e) of avian influenza a virus in plants. Protein Expr Purif. 2007; 56(2):153-9.

22. Reed $\sqcup$, Muench $\mathrm{H}$. A simple method of estimating fifty per cent endpoints. Am J Epidemiol. 1938;27(3):493-7.

23. Denizot F, Lang R. Rapid colorimetric assay for cell growth and survival: modifications to the tetrazolium dye procedure giving improved sensitivity and reliability. J Immunol Methods. 1986;89(2):271-7.

24. Overbergh L, Valckx D, Waer M, Mathieu C. Quantification of murine cytokine mRNAs using real time quantitative reverse transcriptase PCR. Cytokine. 1999;11(4):305-12.

25. Fitzmaurice J, Glennon M, Duffy G, Sheridan J, Carroll C, Maher M. Application of real-time PCR and RT-PCR assays for the detection and quantitation of VT 1 and VT 2 toxin genes in E. coli O157: H7. Mol Cell Probes. 2004;18(2):123-32.

26. Khoufache K, Cabaret O, Farrugia C, Rivollet D, Alliot A, Allaire E, Cordonnier C, Bretagne S, Botterel F. Primary in vitro culture of porcine tracheal epithelial cells in an air-liquid interface as a model to study airway epithelium and aspergillus fumigatus interactions. Med Mycol. 2010;48(8):1049-55.

27. Wulster-Radcliffe MC, Ajuwon KM, Wang J, Christian JA, Spurlock ME. Adiponectin differentially regulates cytokines in porcine macrophages. Biochem Biophys Res Commun. 2004;316(3):924-9.

28. Kiros TG, van Kessel J, Babiuk LA, Gerdts V. Induction, regulation and physiological role of IL-17 secreting helper T-cells isolated from PBMC, thymus, and lung lymphocytes of young pigs. Vet Immunol Immunopathol. 2011;144(3):448-54.

29. Vandesompele J, De Preter K, Pattyn F, Poppe B, Van Roy N, De Paepe A, Speleman F. Accurate normalization of real-time quantitative RT-PCR data by geometric averaging of multiple internal control genes. Genome Biol. 2002;3(7):1-12.

30. Roman M, Martin-Orozco E, Goodman JS, Nguyen MD, Sato Y, Ronaghy A Kornbluth RS, Richman DD, Carson DA, Raz E. Immunostimulatory DNA sequences function as T helper-1-promoting adjuvants. Nat Med. 1997;3(8): 849-54.

31. Rincon M, Anguita J, Nakamura T, Fikrig E, Flavell RA. Interleukin (IL)-6 directs the differentiation of IL-4-producing CD4+ T cells. J Exp Med. 1997; 185(3):461-9.

32. Korn T, Bettelli E, Oukka M, Kuchroo VK. IL-17 and Th17 Cells. Annu Rev Immunol. 2009;27:485-517

33. Korn T, Bettelli E, Gao W, Awasthi A, Jäger A, Strom TB, Oukka M, Kuchroo VK. IL-21 initiates an alternative pathway to induce proinflammatory $\mathrm{TH} 17$ cells. Nature. 2007;448(7152):484-7.

34. Lencer WI, Saslowsky D. Raft trafficking of AB 5 subunit bacterial toxins. Biochim Biophys Acta Mole Cell Res. 2005;1746(3):314-21.

35. Takahara M, Hibler DW, Barr PJ, Gerlt JA, Inouye M. The ompA signal peptide directed secretion of Staphylococcal nuclease A by Escherichia coli. J Biol Chem. 1985;260(5):2670-4.

36. Sato T, Matsui T, Takita E, Kadoyama Y, Makino S, Kato K, Sawada K, Hamabata T. Evaluation of recombinant forms of the shiga toxin variant Stx2eB subunit and non-toxic mutant Stx2e as vaccine candidates against porcine edema disease. J Vet Med Sci. 2013;75(10):1309-15. 
37. Matise I, Cornick NA, Booher SL, Samuel JE, Bosworth BT, Moon HW. Intervention with Shiga toxin (Stx) antibody after infection by Stx-producing escherichia coli. J Infect Dis. 2001;183(2):347-50.

38. McGhee JR, Kiyono H. New perspectives in vaccine development: mucosal immunity to infections. Infect Agents Dis. 1993;2(2):55-73.

39. Anonymous Proceedings of the PEDIATRIC RESEARCH: INT PEDIATRIC RESEARCH FOUNDATION, INC 351 WEST CAMDEN ST, BALTIMORE, MD 21201-2436 USA; 2002

40. Romagnani S. Type $1 \mathrm{~T}$ helper and type $2 \mathrm{~T}$ helper cells: functions, regulation and role in protection and disease. Int J Clin Lab Res. 1992; 21(2-4):152-8.

41. Parham $P$. On the fragmentation of monoclonal $\lg G 1, \lg G 2 a$, and $\lg G 2 b$ from BALB/c mice. J Immunol. 1983;131(6):2895-902.

42. Gupta P, Singh MK, Singh Y, Gautam V, Kumar S, Kumar O, Dhaked RK. Recombinant Shiga toxin B subunit elicits protection against Shiga toxin via mixed Th type immune response in mice. Vaccine. 2011;29(45):8094-100.

43. Hodgkin PD, Lee $J H$, Lyons AB. B cell differentiation and isotype switching is related to division cycle number. J Exp Med. 1996;184(1):277-81.

44. MacLeod MK, Kappler JW, Marrack P. Memory CD4 T cells: generation, reactivation and re-assignment. Immunology. 2010;130(1):10-5.

45. Ren W, Yu R, Liu G, Li N, Peng Y, Wu M, Yin Y, Li Y, Fatufe AA, Li T. DNA vaccine encoding the major virulence factors of Shiga toxin type 2e (Stx2e)expressing escherichia coli induces protection in mice. Vaccine. 2013;31(2): 367-72.

46. Conti HR, Shen F, Nayyar N, Stocum E, Sun JN, Lindemann MJ, Ho AW, Hai $\mathrm{JH}, \mathrm{Yu} J \mathrm{~J}$, Jung JW, Filler SG, Masso-Welch P, Edgerton M, Gaffen SL. Th17 cells and IL-17 receptor signaling are essential for mucosal host defense against oral candidiasis. J Exp Med. 2009;206(2):299-311.

47. Hur J, Lee $\mathrm{JH}$. A new enterotoxigenic escherichia coli vaccine candidate constructed using a Salmonella ghost delivery system: comparative evaluation with a commercial vaccine for neonatal piglet colibacillosis. Vet Immunol Immunopathol. 2015;164(3):101-9.

\section{Submit your next manuscript to BioMed Central and we will help you at every step:}

- We accept pre-submission inquiries

- Our selector tool helps you to find the most relevant journal

- We provide round the clock customer support

- Convenient online submission

- Thorough peer review

- Inclusion in PubMed and all major indexing services

- Maximum visibility for your research

Submit your manuscript at www.biomedcentral.com/submit 